\title{
Effect of Reduced Exercise Frequency After Exercise Intervention on Arterial Stiffness in Middle-Aged and Older Women
}

\author{
H Koshiba $^{1 *}$ and E Maeshima ${ }^{2}$ \\ ${ }^{1}$ Faculty of Health and Well-being, Kansai University, Japan \\ ${ }^{2}$ Graduate School of Health and Sport Sciences, Osaka University of Health and Sport Sciences, Japan \\ *Corresponding author: Hiroya Koshiba, Faculty of Health and Well-being, Kansai University, Japan
}

ARTICLE INFO

Received: 慝 June 06, 2019

Published: 蔧 June 12, 2019

Citation: H Koshiba, E Maeshima. Effect of Reduced Exercise Frequency After Exercise Intervention on Arterial Stiffness in Middle-Aged and Older Women. Biomed J Sci \& Tech Res 18(5)-2019. BJSTR. MS.ID.003208.

Keywords: Arterial Stiffness; Exercise; Pulse Wave Velocity

Abbreviations: BAPWV: Brachial-Ankle Pulse Wave Velocity; NOx: Nitrite/ Nitrate; NO: Nitric Oxide; PWV: Pulse Wave Velocity; FMD: Flow Mediated Dilation

\begin{abstract}
Summary

The present study, by focusing on the effects of exercise, aimed to elucidate how exercising at a reduced frequency after a 12-week exercise intervention would affect arterial stiffness. The present study included 19 middle-aged or older women. After 12 weeks of aerobic exercise, these 19 women were randomly divided into a group of 9 women who would continue exercising once per week for 12 weeks and another group of 10 women who would continue exercising once every 2 weeks for 12 weeks. Measurements were taken three times: before exercise (pre-exercise), 12 weeks after the initiation of exercise $(12 \mathrm{wk})$ and 12 weeks after reduction in the exercise frequency (24wk). As an indicator of arterial stiffness, brachial-ankle pulse wave velocity (baPWV) showed no interactions among the pre-exercise, $12 \mathrm{wk}$, and $24 \mathrm{wk}$ measurement points or between the two groups. However, main effects were observed. Multiple comparison by the Bonferroni method revealed that the $12 \mathrm{wk}$ and $24 \mathrm{wk}$ baPWV was significantly lower than the pre-exercise baPWV. The results of the present study revealed that arterial stiffness could be reduced by exercising for 12 weeks and then maintained low by exercising even at a low frequency of one per week or every 2 weeks.
\end{abstract}

\section{Introduction}

Advancing age is associated with an increase in arterial stiffness [1]. Arterial stiffness has been shown to be an independent risk factor for arteriosclerosis-related diseases [2-5] and a useful prognostic factor in the general population and patients with hypertension or diabetes mellitus [6-9]. Based on these findings, preventing progression of arterial stiffness seems important for the prevention of arteriosclerosis to increase patients' chances of living a healthy, long life. In the 1990s, the Baltimore study demonstrated that exercise is useful for preventing the progression of arterial stiffness [10]. Subsequently, many studies have reported the association between exercise and arterial stiffness, indicating that exercising two to five times per week reduces arterial stiffness [11-17]. Regarding the duration of exercise, even a relatively short duration of exercise intervention, that is 4 to 8 weeks, reportedly leads to a reduction in arterial stiffness $[13,18]$. Furthermore, because exercise intervention results in reduced arterial stiffness and increased nitrite/nitrate (NOx) concentrations [19], nitric oxide (NO) appears to be involved in the mechanism through which exercise reduces arterial stiffness. Moreover, several previous studies have revealed that exercise-induced reduction in arterial stiffness disappears after discontinuation of exercise.

For instance, it has been demonstrated that in people exercising for 8 to 12 weeks, the extent of arterial stiffness returns to preexercise levels 4 to 8 weeks after discontinuing exercise [13,20-22]. Since the effects of exercise on arterial stiffness are reversible, as shown in these studies, continuing exercise is important. In this regard, it has been reported that arterial stiffness increases after discontinuation of exercise in people participating in a short-term 
exercise intervention and in those exercising for a long period [23]. In Japan, people who regularly exercise for 30 minutes or more, twice or more times per week, account for $24.6 \%$ of men aged 20 to 64 years and $19.8 \%$ of women in the same age group. These proportions do not meet the target value set for either men or women (36\% and 33\%, respectively) (Ministry of Health, Labour and Welfare. Japan: The 2015 National Health and Nutrition Survey Overview.). The "lack of time" is reported as a reason for not exercising by $50.7 \%$ of the surveyed Japanese individuals (Ministry of Education, Culture, Sports, Science and Technology. Japan: National Survey of Participants in Sports and Physical Activities). Thus, if the effects obtained by exercise can be maintained by exercising at a lower frequency, the number of people who exercise can be increased in Japan. The present study, by focusing on the effects of exercise, aimed to elucidate how exercising at a reduced frequency after a 12-week exercise intervention would affect arterial stiffness. In addition, the association between arterial stiffness and the NO concentration after reduction in the exercise frequency was also investigated.

\section{Methods}

\section{Subjects}

The present study included 19 middle-aged or older women (61.1 \pm 1.6 years) without either exercise or smoking habits. Furthermore, it was ensured that no women receiving either oral contraceptives or hormone replacement therapy were included. After 12 weeks of aerobic exercise, these 19 women were randomly divided into a group of 9 women who would continue exercising once per week for 12 weeks (once/week group) and another group of 10 women who would continue exercising once every 2 weeks for 12 weeks (once/2wk group). Measurements were taken three times: before exercise (pre-exercise), 12 weeks after the initiation of exercise $(12 \mathrm{wk})$, and 12 weeks after reduction in the exercise frequency (24wk). Written and verbal explanations of the objectives, methods, and safety of the present study were provided to the participants. After they sufficiently understood the contents of the study, written informed consent was obtained. This study was approved as a "research on humans" by the ethics committee of Osaka University of Health and Sport Sciences (approval no. 14-35).

\section{Measurements}

For body composition, height, weight, body mass index, and body fat percentage were measured with the Body Composition Analyzer In Body 3.2 (Biospace Co., Ltd., Tokyo, Japan). As an indicator of arterial stiffness, brachial-ankle pulse wave velocity (baPWV) was measured with the formPWV/ABI@ (Omron Colin Co., Ltd., Tokyo, Japan). Simultaneously, systolic and diastolic blood pressures were measured with this device. The baPWV is a pulse wave velocity (PWV) calculated from the time interval between pulse waves of the arms and ankle joints detected with cuffs for measuring blood pressure and the vessel length (distance calculated by subtracting the vessel length of the aortic orifice-to-brachial segment from the vessel length of the aortic orifice-to-ankle segment). Since
baPWV is determined by measuring the PWV between the brachial and fibular arteries, it reflects PWV measurements of elastic and muscular arteries, such as the aorta. However, baPWV is highly correlated with aortic PWV measured by catheterization, easy to measure, and highly reproducible; therefore, it is widely used as a clinical indicator of arteriosclerosis in Japan [24].

The formPWV/ABI ${ }^{\circ}$ generally yields a mean value during one or more respiratory cycles because it measures pulse waves for 10 seconds and averages the measurements. The mean of the right and left baPWV was used as a representative value for each participant in the analyses. Arterial stiffness and blood pressures were measured 3 hours or more after breakfast, while the participants were prohibited from performing vigorous exercise for at least 12 hours before the measurements and consuming caffeinated drinks after arising in the morning. In a room maintained at about $24^{\circ} \mathrm{C}$, the measurements were obtained after 15 minutes of rest. As an indicator of NO, blood NOx concentrations were measured using high-performance liquid chromatography [25]. While the participants had been instructed not to consume anything except water after 21:00 on the day before the test, blood specimens were collected from the cubital vein after fasting for 12 hours or more. Analyses of specimens were outsourced to Japan Clinical Laboratories, Inc. (Hyogo, Japan).

\section{Aerobic Exercise Intervention}

Participants underwent an aerobic exercise intervention consisting of walking and bicycle ergometer exercise (Aerobike 75XL II or Aerobike 75 XL III, Combi Corporation, Tokyo, Japan) at an intensity of $50 \%$ heart rate reserve for 50 minutes, two to three times per week (twice per week under supervision) for 12 weeks. Then, the participants were randomly divided into the once/week group of those exercising once per week and the once/2wk group of those exercising once every 2 weeks and continuing aerobic exercise with the bicycle ergometer at the same intensity for the same duration of each session for 12 weeks.

\section{Statistical Analyses}

In the present study, all results are expressed as a mean \pm standard error. Two-way analysis of variance (group $\times$ time) was performed for comparison between the once/week and once/2wk groups (group) and among the pre-exercise, $12 \mathrm{wk}$, and $24 \mathrm{wk}$ values (time). When the analysis of variance showed significant differences, a significance test was performed using the Bonferroni method. Associations between the parameters were analyzed with Pearson product-moment correlation coefficients. SPSS (version 21.0, SPSS Japan Inc., Tokyo, Japan) was used for statistical analyses, and the statistical significance level was set to less than $5 \%$.

\section{Results}

\section{Physical Examination, Blood Pressure and NO}

Table 1 shows the characteristics of participants at the pre-exercise, $12 \mathrm{wk}$, and $24 \mathrm{wk}$ measurement points. Although no interac- 
tions were observed for any indicators, main effects were observed for age at the pre-exercise, $12 \mathrm{wk}$, and $24 \mathrm{wk}$ measurement points. Multiple comparison using the Bonferroni method revealed that age was significantly higher at the $24 \mathrm{wk}$ measurement point than at the pre-exercise measurement point $(\mathrm{p}<0.05)$. Blood pressure showed no interactions among the pre-exercise, $12 \mathrm{wk}$, and $24 \mathrm{wk}$ measurement points or between the two groups. However, main effects were observed for only systolic blood pressure at the pre-exercise, $12 \mathrm{wk}$, and $24 \mathrm{wk}$ measurement points. Multiple comparison using the Bonferroni method revealed that systolic blood pressure was significantly lower at the $12 \mathrm{wk}$ measurement point than at the pre-exercise measurement point ( $\mathrm{p}<0.05$ ). Blood NOx concentrations, which were measured as an indicator of NO, showed neither interactions nor main effects among the pre-exercise, $12 \mathrm{wk}$, and $24 \mathrm{wk}$ measurement points or between the two groups.

\section{Arterial Stiffness}

The baPWV showed no interactions among the pre-exercise, $12 \mathrm{wk}$, and $24 \mathrm{wk}$ measurement points or between the two groups. However, main effects were observed for the pre-exercise, $12 \mathrm{wk}$, and 24wk baPWV. Multiple comparison by the Bonferroni method revealed that the $12 \mathrm{wk}$ and $24 \mathrm{wk}$ baPWV was significantly lower than the pre-exercise baPWV (p <0.05) (Figure 1).

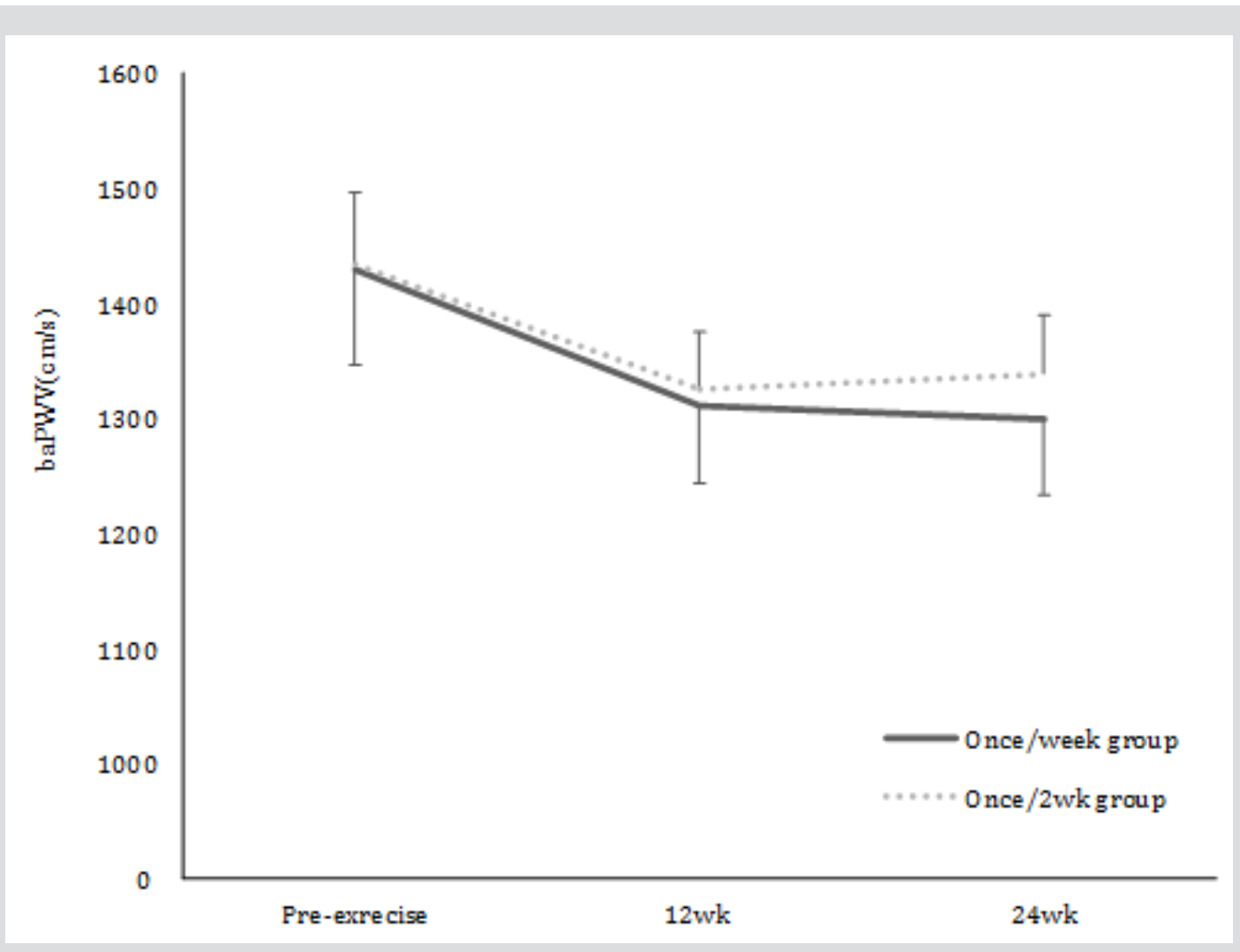

Figure 1: Time course of arterial stiffness. Main effects were observed in pre, $12 \mathrm{wk}, 24 \mathrm{wk}$ of baPWV. A significant reduction in baPWV was observed at $12 \mathrm{wk}$ and $24 \mathrm{wk}$ compared with pre-by multiple comparison.

\section{Association Between Arterial Stiffness and NO}

Blood NOx concentrations, which were measured as an indicator of NO, showed neither interactions nor main effects among the pre-exercise, $12 \mathrm{wk}$, and $24 \mathrm{wk}$ measurement points or between the two groups (Table 1). When the association between percentage changes in arterial stiffness and NO concentrations was assessed, the percentage changes in baPWV from the pre-exercise to $24 w k$ measurement point were no correlated with the percentage changes in blood NOx concentrations during the same period in the overall sample. However, the once/week group showed correlation between percentage changes in baPWV and blood NOx concentrations from the pre-exercise to $24 \mathrm{wk}$ measurement points. In other words, as baPWV decreased, blood NOx concentrations increased (Table 2). By contrast, the once/2wk group showed no correlation between percentage changes in baPWV and blood NOx concentrations from the pre-exercise to $24 \mathrm{wk}$ measurement points (Table 2). 
Table 1: Characteristics of Subjects.

\begin{tabular}{|c|c|c|c|c|c|c|}
\hline & \multicolumn{3}{|c|}{ Once/week Group } & \multicolumn{3}{c|}{ Once/2wk Group } \\
\hline & Pre-Exercise & $\mathbf{1 2 w k}$ & $\mathbf{2 4 w k}$ & Pre-Exercise & $\mathbf{1 2 w k}$ & $\mathbf{2 4 w k}$ \\
\hline Age (Years) & $61.9 \pm 2.5$ & $62.1 \pm 2.6$ & $62.4 \pm 2.5$ & $59.4 \pm 2.1$ & $59.8 \pm 2.1$ & $59.8 \pm 2.1$ \\
\hline Height (cm) & $157.8 \pm 2.1$ & - & - & $153.3 \pm .0 .6$ & - & - \\
\hline Body Mass(Kg) & $59.6 \pm 3.3$ & $58.7 \pm 3.0$ & $58.7 \pm 3.1$ & $52.9 \pm 1.7$ & $52.5 \pm 1.7$ & $52.5 \pm 1.8$ \\
\hline BMI ( kg/m²) & $23.9 \pm 1.0$ & $23.5 \pm 0.9$ & $23.2 \pm 1.0$ & $22.5 \pm 06$ & $22.3 \pm 06$ & $22.3 \pm 0.7$ \\
\hline Body Fat (\%) & $29.8 \pm 1.5$ & $29.7 \pm 1.1$ & $29.4 \pm 1.3$ & $29.3 \pm 1.4$ & $28.9 \pm 1.5$ & $29.1 \pm 1.4$ \\
\hline SBP (mmHg) & $122.8 \pm 3.8$ & $113.1 \pm 3.0$ & $113.9 \pm 2.9$ & $130.2 \pm 6.3$ & $120.7 \pm 5.3$ & $123.7 \pm 4.3$ \\
\hline DBP (mmHg) & $72.6 \pm 3.0$ & $68.1 \pm 3.1$ & $69.2 \pm 2.8$ & $69.2 \pm 2.8$ & $74.1 \pm 2.0$ & $75.2 \pm 1.9$ \\
\hline
\end{tabular}

Note: Values are mean \pm SEM. 12wk: 12 weeks after the initiation of exercise, $24 \mathrm{wk}$ : 12 weeks after reduction in the exercise frequency, BMI: Body Mass index, SBP: systolic blood pressure, DBP: diastolic blood pressure.

Table 2: Correlation between percentage changes in arterial stiffness and NO.

\begin{tabular}{|c|c|c|c|c|c|c|}
\hline & \multicolumn{2}{|c|}{ All (n=19) } & \multicolumn{2}{c|}{ Once/Week(n=9) } & \multicolumn{2}{c|}{ Once/2wk(n=10) } \\
\hline & $\mathbf{r}$ & p-value & $\mathbf{r}$ & $\mathbf{p}$-value & $\mathbf{r}$ & $\mathbf{p}$-value \\
\hline$\Delta_{\text {NOx }}$ & -0.342 & 0.152 & -0.670 & $0.048^{*}$ & -0.94 & 0.795 \\
\hline
\end{tabular}

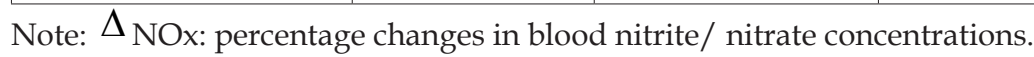
${ }^{*} \mathrm{p}<0.05$.

\section{Discussion}

We revealed that after baPWV was reduced by 12 weeks of exercise interventions, it could be maintained low by continuing exercise once per week or every 2 weeks. The effects obtained by exercise vary depending on variables, such as modes, frequency, duration of each session, and duration of the entire course of exercise. These variables need to be adjusted according to the objectives of exercise, such as reduction in arterial stiffness or blood pressure. In the present study, participants performed aerobic exercise for 50 minutes, two to three times per week for 12 weeks to reduce arterial stiffness, based on previous studies [11-17,26,27]. As a result, arterial stiffness and systolic blood pressure decreased after exercising for 12 weeks. A previous study indicated that exercising for 30 to 60 minutes per week exerts a hypotensive effect on systolic blood pressure [28]. In the present study, because the duration of exercise per week ranged from 100 to 150 minutes, we could determine that our participants exercised long enough to reduce blood pressure. For these reasons, we assume that arterial stiffness and systolic blood pressure decreased in the present study. However, after the exercise frequency was reduced, the duration of exercise in the once/2wk group was not enough to reduce blood pressure; consequently, the hypotensive effect of exercise was not maintained. Thus, exercising for 50 minutes every 2 weeks was found to be insufficient, in terms of the quantity of exercise, to reduce blood pressure and to maintain it at low levels achieved by exercise.

We set the exercise frequency to once per week and once every 2 weeks in the setting of maintaining reduced arterial stiffness. Previous studies have shown no consensus on whether exercising once per week reduces arterial stiffness. For instance, Shin et al. reported that continuing Tai Chi training once per week reduces arterial stiffness [29]. In contrast, Miura et al. reported that arterial stiffness is not reduced by continuing exercise once per week [30]. Thus, we set the exercise frequency to once per week in the setting of maintaining reduced arterial stiffness and compared this frequency with a half frequency of exercising once every 2 weeks. As a result, we found that arterial stiffness reduced by exercise could be maintained low by exercising once per week and once every 2 weeks. Based on previous studies, reduction achieved by 12 weeks of exercise interventions in arterial stiffness will disappear in 4 weeks without continuing exercise [21]. In the present study, because the participants continued exercise during this period of 4 weeks when the reducing effect on arterial stiffness disappears, baPWV, which is an indicator of arterial stiffness, was presumably maintained low. This view can be tested in a previous study conducted by Zheng et al. [31]. They assessed the effects of temporary exercise on arterial stiffness and reported that reduction achieved by one session of exercise in arterial stiffness does not last for 60 minutes after the end of exercise. However, they demonstrated that when participants exercised twice with an interval of 20 minutes, reduction in arterial stiffness after the second session of exercise lasted for more than 60 minutes after the end of exercise. This suggests that repeating exercise at an appropriate timing prolongs the effect of exercise on arterial stiffness. The appropriate timing may be at 20 minutes after temporary exercise in the study by Zheng et al. [31] and at 1 to 2 weeks after exercise in our study. Thus, we consider that the results of our study support the study by Zheng et al. However, the mechanism through which arterial stiffness was maintained low 
after reduction in the exercise frequency remains unknown. We assume that NO underlies this mechanism. Since arterial stiffness was reduced by exercise interventions in a relatively short period of 12 weeks, changes in the composition of vessel wall components, such as calcium and collagen, are less likely to have contributed to reduced arterial stiffness. Instead, the tonicity of vascular smooth muscle is highly likely to have been changed through the vascular endothelial function, including the NO concentration. In fact, Shin et al. reported that the decreased rate of arterial stiffness was significantly correlated with that of flow mediated dilation (FMD) in participants exercising once per week for 3 months [29]. FMD is an indicator for assessing NO like NOx, which was used in the present study. Furthermore, exercise-induced improvement in FMD is strongly correlated with exercise-induced improvement in NOx concentrations ( $\mathrm{r}=0.81$ ) [32].

The present study also revealed that the percentage change in arterial stiffness was significantly correlated with the percentage change in NOx concentrations only in the once/week group. This suggests that NO may be greatly involved in maintaining low arterial stiffness by exercising once per week. However, NO was not associated with a reduction in arterial stiffness in the once/2wk group. In previous studies, exercise-induced improvement in $\mathrm{NOx}$ concentrations was observed in participants exercising once per week or three to four times per week $[19,29,33]$. This suggests that substances other than NO may also be involved in maintaining low arterial stiffness by exercising once every 2 weeks. The blood NOx concentration is a simple and less invasive indicator of vascular endothelial function. However, because it is an indirect indicator for measuring levels of NO metabolites [34], it is unclear to what extent this indicator can account for the association between arterial stiffness after reduction in the exercise frequency and NO in the present study. This is a limitation of the study. In the future, more detailed studies are warranted to elucidate the mechanisms regarding the duration of effects of exercise on arterial stiffness.

\section{Conclusion}

The present study revealed that arterial stiffness could be reduced by exercising for 12 weeks and then maintained low by exercising even at a low frequency of one per week or every 2 weeks. In addition, it has been suggested that NO might be involved in maintaining low arterial stiffness by exercising once per week. The present findings may have important implications for designing more acceptable exercise prescription, for the arterial stiffness improvement.

\section{Acknowledgment}

we would like to thank Yuka Okumura for your valuable contribution in data collection. We also thank all the participants for their time and commitment. This study was partially supported by a grant in aid from National Strength and Conditioning Association Japan.

\section{Conflict of Interest}

No conflicts of interest, financial or otherwise, are declared by the authors.

\section{References}

1. Tomiyama H, Yamashina A, Arai T, Hirose K, Koji Y, et al. (2003) Influences of age and gender on results of noninvasive brachial-ankle pulse wave velocity measurement-a survey of 1257 subjects. Atherosclerosis 166(2): 303-309.

2. Benetos A, Adamopoulos C, Bureau JM, Temmar M, Labat C, et al. (2002) Determinants of accelerated progression of arterial stiffness in normotensive subjects and in treated hypertensive subjects over a 6-year period. Circulation 105(10): 1202-1207.

3. Boutouyrie P, Tropeano AI, Asmar R, Gautier I, Benetos A, et al. (2002) Aortic stiffness is an independent predictor of primary coronary events in hypertensive patients: a longitudinal study. Hypertension 39(1): 10-15.

4. Laurent S, Katsahian S, Fassot C, Tropeano AI, Gautier I, et al. (2003) Aortic stiffness is an independent predictor of fatal stroke in essential hypertension. Stroke 34(5): 1203-1206.

5. Mattace-Raso FU, van der Cammen TJ, Hofman A, van Popele NM, Bos ML, et al. (2006) Arterial stiffness and risk of coronary heart disease and stroke: the Rotterdam Study. Circulation 113(5): 657-663.

6. Cruickshank K, Riste L, Anderson SG, Wright JS, Dunn G, et al. (2002) Aortic pulse-wave velocity and its relationship to mortality in diabetes and glucose intolerance: an integrated index of vascular function? Circulation 106(16): 2085-2090.

7. Inoue N, Maeda R, Kawakami H, Shokawa T, Yamamoto H, et al. (2009) Aortic pulse wave velocity predicts cardiovascular mortality in middleaged and elderly Japanese men. Circ J 73(3): 549-553.

8. Laurent S, Boutouyrie P, Asmar R, Gautier I, Laloux B, et al. (2001) Aortic stiffness isan independent predictor of all-cause and cardiovascular mortality in hypertensive patients. Hypertension 37(5): 1236-1241.

9. Turin TC, Kita Y, Rumana N, Takashima N, Kadota A, et al. (2010) Brachialankle pulse wave velocity predicts all-cause mortality in the general population: findings from the Takashima study, Japan. Hypertens Res 33(9): 922-925.

10. Vaitkevicius PV, Fleg JL, Engel JH, O'Connor FC, Wright JG, et al. (1993) Effects of age and aerobic capacity on arterial stiffness in healty adults. Circulation 88(4): 1456-1462.

11. Fantin F, Rossi A, Morgante S, Soave D, Bissoli L, et al. (2012) Supervised walking groups to increase physical activity in elderly women with and without hypertension: effect on pulse wave velocity. Hypertens Res 35(10): 988-993.

12. Hayashi K, Sugawara J, Komine H, Maeda S, Yokoi T (2005) Effects of aerobic exercise training on the stiffness of central and peripheral arteries in middle-age sedentary men. Jpn J Physiol 55(4): 235-239.

13. Kakiyama T, Sugawara J, Murakami H, Maeda S, Kuno S, et al. (2005) Effects of short-term endurance training on aortic distensibility in young males. Med Sci Sports Exerc 37(2) 267-271.

14. Madden KM, Lockhart C, Cuff D, Potter TF, Meneilly GS (2013) Aerobic training-induced improvements in arterial stiffness are not sustained in older adults with multiple cardiovascular risk factors. J Hum Hypertens 27(5): 335-339.

15. McNeilly AM, McClean C, Murphy M, McEneny J, Trinick T, et al. (2012) Exercise training and impaired glucose tolerance in obese humans. J Sports Sci 30(8): 725-732.

16. Millen AM, Norton GR, Avidon I, Woodiwiss AJ (2013) Effects of shortterm exercise-training on aortic systolic pressure augmentation in overweight and obese individuals. Eur J Appl Physiol 113(7):1793-1803. 
17. Sugawara J, Otsuki T, Tanabe T, Hayashi K, Maeda S, et al. (2006) Physical activity duration, intensity, and arterial stiffening in postmenopausal women. Am J Hypertens 19(10): 1032-1036.

18. Cameron JD, Dart AM (1994) Exercise training increases total systemic arterial compliance in humans. Am J Physiol 266(2): 693-701.

19. Fujie S, Sato K, Miyamoto Mikami E, Hasegawa N, Fujita S, et al. (2014) Reduction of arterial stiffness by exercise training is associated with increasing plasma apelin level in middle-aged and older adults. Plos one 9(4): 93545.

20. Madhura M, Sandhya TA (2015) Effect of short duration aerobic exercise training on reflection index, stiffness index and pulse wave velocity. Indian J physiol Pharmacol 56(1): 15-20.

21. Mustata S, Chan C, Lai V, Miller JA (2004) Impact of an exercise program on arterial stiffness and insulin resistance in hemodialysis patients. J Am Soc Nephrol 15(10): 2713-2718.

22. Okamoto T, Masuhara M, Ikuta K (2007) Combined aerobic and resistance training and vascular function: effect of aerobic exercise before and after resistance training. J Appl Physiol 103(5): 1655-1661.

23. Koshiba H, Maeshima Etsuko (2015) Influence of detraining on temporal changes in arterial stiffness in endurance athletes: a prospective study. J Phys Ther Sci 27(12): 3681-3684.

24. Yamashina A, Tomiyama H, Takeda K, Tsuda H, Arai T, et al. (2002) Validity, reproducibility, and clinical significance of noninvasive brachialankle pulse wave velocity measurement. hypertens Res 25(3): 359-364

25. Green LC, Wagner DA, Glogowski J, Skipper PL, Wishnok JS, et al. (1982) Analysis of nitrate, nitrite, and [15N] nitrate in biological fluids. Anal Biochem 126(1): 131-138.

26. Ashor AW, Lara J, Siervo M, Celis-Morales C, Mathers JC (2014) Effects of exercise modalities on arterial stiffness and wave reflection: a systematic

\section{ISSN: 2574-1241}

DOI: 10.26717/BJSTR.2019.18.003208

Hiroya Koshiba. Biomed J Sci \& Tech Res

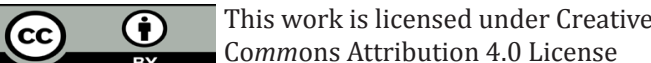

Submission Link: https://biomedres.us/submit-manuscript.php review and meta-analysis of randomized controlled trials. Plos one 9: 110034

27. Montero D, Vinet A, Roberts CK (2015) Effect of combined aerobic and resistance training versus aerobic training on arterial stiffness. Int Cardiol 178: 69-76.

28. Ishikawa-Takata K, Ohta T, Tanaka H (2003) How much exercise is required to reduce blood pressure in essential hypertensives: a doseresponse study. Am J Hypertens 16(8): 629-633.

29. Shin JH, Lee Y, Kim SG, Choi BY, Lee HS, et al. (2015) The beneficial effects of Tai Chi exercise onendothelial function and arterial stiffness in elderly women with rheumatoid arthritis. Arthritis Res Ther 17: 380.

30. Miura H, Nakagawa E, Takahashi Y (2008) Influence of group training frequency on arterial stiffness in elderly women. Eur J Appl Physiol 104(6): 1039-1044

31. Zheng L, Zhang X, Zhu W, Chen X, Wu H, et al. (2015) Acute effects of moderate-intensity continuous and accumulated exercise on arterial stiffness in healthy young men. Eur J Appl Physiol 115(1): 177-185.

32. Steiner S, Niessner A, Ziegler S, Richter B, Seidinger D, et al. (2005) Endurance training increases the number of endothelial progenitor cells in patients with cardiovascular risk and coronary artery disease. Atherosclerosis 181(2): 305-310.

33. Maeda S, Miyauchi T, Kakiyama T, Sugawara J, Iemitsu M, et al. ( 2001) Effects of exercise training of 8 weeks and detraining on plasma levels of endothelium-derived factors, endothelin-1 and nitric oxide, in healthy young humans. Life Sci 69(9): 1005-1016.

34. Lind L, Hall J, Larsson A, Annuk M, Fellström B, et al. (2000) Evaluation of endothelium-dependent vasodilation in the human peripheral circulation. Clin Physiol 20(6): 440-448.

$\begin{array}{ll}\text { BIOMEDICAL } & \text { Assets of Publishing with us } \\ \text { RESEARCHES } & \text { - Global archiving of articles } \\ & \text { - Immediate, unrestricted online access } \\ & \text { - Rigorous Peer Review Process } \\ \end{array}$

